\title{
The Impact of Comorbid Depression on Adherence to Therapy for Multiple Sclerosis
}

\author{
M. Tarrants, ${ }^{1}$ M. Oleen-Burkey, ${ }^{1}$ J. Castelli-Haley, ${ }^{1}$ and M. J. Lage ${ }^{2}$ \\ ${ }^{1}$ Medical Affairs, Teva Pharmaceuticals, 901 East 104th Street, Suite 900, Kansas City, MO 64131, USA \\ ${ }^{2}$ HealthMetrics Outcomes Research, 120 Anchorage Circle, Groton, CT 06340, USA \\ Correspondence should be addressed to M. J. Lage, lagemj@hlthmetrics.com
}

Received 3 November 2010; Revised 7 March 2011; Accepted 29 April 2011

Academic Editor: Peter Arnett

Copyright (c) 2011 M. Tarrants et al. This is an open access article distributed under the Creative Commons Attribution License, which permits unrestricted use, distribution, and reproduction in any medium, provided the original work is properly cited.

\begin{abstract}
Objective. Examine the impact of comorbid depression on adherence to disease-modifying therapy (DMT) for multiple sclerosis (MS). Methods. A retrospective database was used to identify patients with MS treated with a DMT. Patients with MS and comorbid depression were matched to patients with MS only. Adherence to DMT was proxied by the medication possession ratio (MPR) and multivariate regressions were used to examine the association between comorbid depression and adherence to DMT. Results. Patients with comorbid depression had a 10 point lower MPR $(P<0.01)$ and were less likely to achieve a MPR of at least $80 \%$ (odds ratio $(\mathrm{OR})=0.55 ; 95 \%$ confidence interval $(\mathrm{CI}) 0.42-0.74)$ than those without depression. While treatment with an antidepressant generally had no significant impact on the likelihood of achieving an MPR threshold of $80 \%(\mathrm{OR}=1.32$; 95\% CI 0.50-3.48), adherence to antidepressant therapy guidelines were associated with improved adherence to DMT therapy. Conclusions. MS patients with comorbid depression were approximately half as likely to be adherent to their DMT relative to patients with MS without depression. Although treatment with antidepressant therapy generally did not improve the likelihood of adherence, treatment with antidepressants for at least 6 months was associated with better adherence to DMT.
\end{abstract}

\section{Introduction}

Multiple sclerosis (MS) is the most common disabling neurological condition for young adults and adolescents in the United States [1]. Approximately 400,000 Americans have MS, and every week about 200 people are diagnosed with the disease [2]. While the results of the existing burden-of-illness studies conducted for MS in the United States vary according to the data sources used [3], they all report substantial economic impacts of MS, both to the individual and to the nation. The cost of each MS relapse has been reported to be approximately $\$ 12,870$ [4]. The average cost of MS for the individual patient, each year, is anywhere from $\$ 12,879$ (2004 USD) [5] to $\$ 34,000$ (1994 USD) [6]. These figures translate to a national cost of $\$ 5.2$ billion $(\$ 12,879 \times 400,000$ Americans diagnosed with MS) [5] to $\$ 6.8$ billion (based on prevalence figures used by the authors of [6], annually.

While MS is incurable, Food and Drug Administration(FDA-) approved disease-modifying therapies (DMTs) have been shown to reduce the rate of relapse and to slow disease progression $[7,8]$. However, medication can only work if it is taken. Lack of medication adherence has been shown to be associated with increased patient morbidity, poorer quality of life, and increased financial strain on health care institutions [9]. In addition, research has also shown that adherence is generally suboptimal among patients with MS [10-13].

One factor that has been shown to negatively impact adherence among patients with MS is the presence of comorbid depression $[10,13]$. This factor may affect a large portion of the MS population, since depression is common among individuals with MS. Research has shown that up to onehalf of all MS patients [14, 15] have comorbid depression, and that the prevalence of this comorbidity is three-times the rate of major depression and psychiatric comorbidity in community-based samples and greater than the rate of depression among patients with other neurological disorders [16].

Given the potential impact of depression on MS adherence as well as the relatively large prevalence of this comorbidity, this analysis was designed to quantify the impact of 
comorbid depression and antidepressant therapy on adherence to disease-modifying therapy (DMT) for MS. Given the previous literature reporting a connection between mood disorders and adherence among patients with MS [10], we hypothesized that a diagnosis of comorbid depression would have a negative impact on adherence. In addition, we examined the impact of receipt of antidepressant therapy on adherence to MS adherence. In so doing, the analysis quantifies the association between comorbid depression, receipt of antidepressants, and adherence to MS therapy.

\section{Materials and Methods}

2.1. Data. The MedStat MarketScan Commercial Claims and Encounters (CCAE) and Medicare (MDCR) databases provided the data for these analyses, which focused exclusively on data from January 1, 2004 through March 25, 2009. These retrospective insurance claims databases include private sector health data from approximately 100 payers and contain data on clinical utilization, expenditures, and enrollment across inpatient, outpatient, prescription drug, and carve-out services. The deidentified databases are fully Health Insurance Portability and Accountability Act (HIPAA) compliant and link paid claims and encounter data to detailed patient information across sites and types of providers over time.

2.2. Sample. To be considered for inclusion in the study, patients had to have received at least one diagnosis of MS (ICD-9-CM code of 340.xx) and a disease modifying therapy (DMT), with the first such receipt in the date window identified as the index date and the initial DMT received identified as the intent-to-treat (ITT) medication. Patients were excluded if they were diagnosed with pregnancy (ICD9-CM code of 630.xx-676.xx, V22.2x, or V22.3x) over the time period from 6 months prior to the index date (i.e., the pre-period) through 12 months after index date (i.e., the postperiod). Finally, patients were required to have continuous insurance coverage from the start of the preperiod through the end of the post-period. These criteria resulted in a sample of 11,780 individuals (Figure 1).

Given the above cohort of individuals, the group was then subdivided into patients who had neither a diagnosis of depression nor the receipt of an antidepressant in the pre-period (i.e., MS Only) and those who were identified with comorbid depression (i.e., MS \& Depression). To be identified as having depression in the 6 month pre-period, patients were required to receive 2 or more outpatient or 1 or more inpatient diagnoses of depression (ICD-9CM of 296.2x, 296.3x, or 311.xx), or 1 or more diagnoses of depression and 1 or more filled prescriptions for an antidepressant. For patients who did not receive an antidepressant, the requirement of one inpatient diagnosis or two outpatient diagnoses of depression was utilized to insure that a patient was diagnosed with depression and not simply screened for depression. Furthermore, such an algorithm is commonly used to identify diagnoses in retrospective databases $[17,18]$. There were 4,479 individuals diagnosed with MS who had no evidence of depression (MS Only) and 448 individuals identified as having MS and comorbid depression (MS \& Depression). Of the 448 individuals with MS and Depression, 379 individuals received at least one prescription for an antidepressant.

Given the above sample of patients, three subgroups of patients were examined. One group consisted of a matched group of patients with MS only to those with MS and depression, where patients were matched $1: 1$ without replacement based upon age, sex, region of residence, year of index date, and intent-to-treat medication. This matching resulted in a successful match rate of $97 \%$ and a final sample of 876 individuals: 436 individuals with MS and depression and 436 individuals with MS only. In addition to examining patients with MS only to those with MS and depression, we also examined adherence by comparing patients who were diagnosed with depression but did not receive an antidepressant $(N=59)$ to those diagnosed with depression who did receive an antidepressant $(N=359)$. These two groups were matched $1: 1$ without replacement using the same factors used to match the MS Only group to the MS \& Depression group. This process resulted in a final sample of 76 individuals: 38 individuals with a diagnosis of depression and no receipt of an antidepressant and 38 individuals who were both diagnosed with depression and filled at least one prescription for an antidepressant. The third group compared patients who received an antidepressant based upon adherence to treatment guidelines for use of antidepressants [19]. Specifically, patients were divided based upon receipt of at least a 6-month supply of antidepressants. Again, the two cohorts were matched $1: 1$ without replacement, with a final sample of 142 individuals: 71 treated according to guidelines, and 71 who received less than 6 months of antidepressants.

2.3. Measuring Adherence. While adherence generally measures the extent to which a patient acts in accordance with the prescribed interval and dose of a dosing regimen [20], such information is not typically available in a retrospective claims database. As such, adherence is "...operationalized in retrospective assessments as the number of doses dispensed in relation to the dispensing period, often called the medication possession ratio (MPR)" [20]. This variable is measured as the percentage of days the ITT medication was available to the patient based on prescription fill dates over the 1-year post-period, and is a common proxy for adherence when examining retrospective data $[6,21,22]$. As a test of the robustness of results, we also considered an alternative proxy for adherence. Specifically, for each patient the variable proportion of days covered (PDC) was also constructed [23]. The PDC, like the MPR, is a measure of the percentage of days a person received their ITT drug over the year after index date. However, in contrast to the MPR, the PDC does not allow for prescriptions to overlap. As a result, the PDC, unlike the MPR, will always have an upper bound of 1 . The results reported here are not sensitive to this alternative specification and hence, results where PDC was used as the dependent variable were not reported. It should be noted, however, that neither the MPR nor PDC can measure whether an individual took a prescription once filled, whether an individual filled a prescription that was 


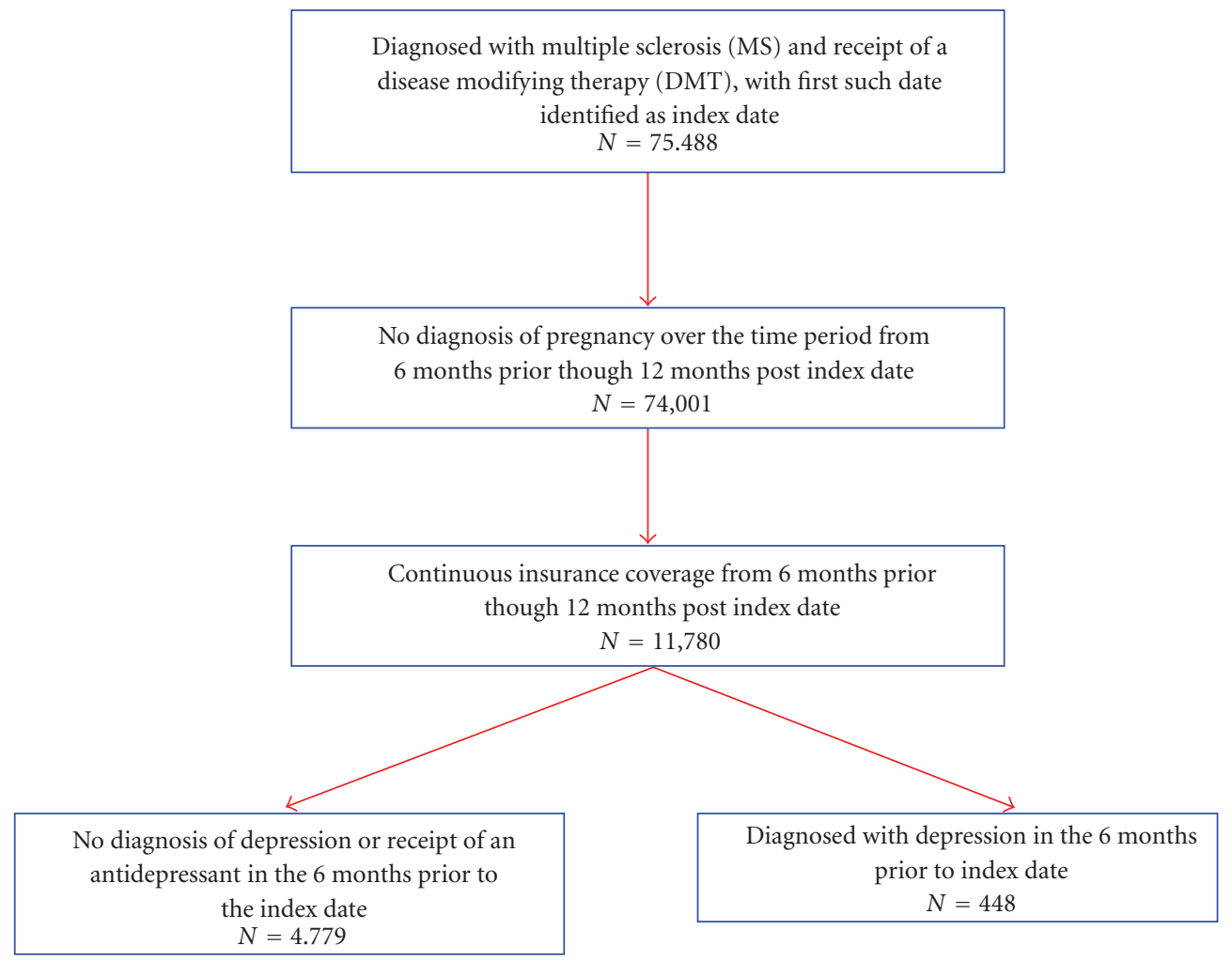

Figure 1: Inclusion/exclusion criteria.

written by their physician, or whether the individual stopped their ITT medication on or against physician advice.

\subsection{Statistical Analyses. Descriptive statistics were compared} between groups using chi-square statistics. Multivariate regressions were used to examine the impact of comorbid depression as well as medical treatment for depression on the likelihood of a patient being adherent to their MS ITT DMT. Specifically, ordinary least squares regressions were used to examine the impact of depression on MPR when MPR was measured as a continuous variables, while logistic models were used to examine the association of depression on the probability of achieving an MPR threshold of at least $80 \%$ $[24,25]$.

In multivariate models it was hypothesized that, in addition to the factors that groups were matched on (age, sex, region of residence, ITT DMT therapy, and year of index date), other patient characteristics might also affect adherence. Specifically, these analyses also examined the association between patient insurance status, characteristics of ITT medication, patient general health, and patient disability level and adherence to ITT DMT. Characteristics of the ITT medication that were examined were the amount of copayment the individual was responsible for as well as whether the ITT medication was delivered via mail order. It was hypothesized that patients required to make a copayment for their medication may be less adherent to their medication regimen while mail order delivery may be associated with ease of use, and therefore may be associated with improved adherence. Patient general health status was proxied by the Charlson Comorbidity Index (CCI) [26, 27]. This measure, which is well validated as an algorithm for predicting hospital mortality and is often used in retrospective database analyses, is constructed of a weighted average based upon the receipt of diagnostic codes associated with 17 medical conditions. Examples of conditions included in the CCI include renal disease, myocardial infarction, malignancy, liver disease, cerebrovascular disease, congestive heart failure, dementia, and diabetes [28]. Finally, patient level of disability was proxied by the presence of musculoskeletal or medical disabilities. These disabilities were proxied by examining the presence of diagnoses for secondary conditions associated with muculoskeletal or medical disabilities, and prior research has shown that these proxies are associated with both the presence and severity of disabilities [29].

All analyses were conducted using SAS version 9.3. Findings of $P$ values $\leq 0.05$ were considered to indicate statistically significant results.

\section{Results and Discussion}

3.1. Results: Descriptive Statistics. As expected, in all cases, groups were identical in terms of the matching variables age, sex, region of residence, ITT DMT, and year of index date. When comparing the MS only group to patients with MS and depression (Table 1), results revealed that patients diagnosed with comorbid depression were significantly less likely to be insured via point-of-service insurance (13.30\% versus 
TABLE 1: Patient characteristics: patients with MS Only compared to Patients with MS \& Depression.

\begin{tabular}{|c|c|c|c|c|c|c|}
\hline \multirow{2}{*}{ Characteristic } & \multicolumn{2}{|c|}{ MS Only $(N=436)$} & \multicolumn{2}{|c|}{ MS \& Depression $(N=436)$} & \multirow{2}{*}{$\chi^{2}$ statistic } & \multirow{2}{*}{$P$ value } \\
\hline & $N$ & $\%$ & $N$ & $\%$ & & \\
\hline \multicolumn{7}{|l|}{ Characteristics matched on } \\
\hline Age & & & & & N/A & N/A \\
\hline$<35$ & 54 & 12.39 & 54 & 12.39 & & \\
\hline $35-44$ & 107 & 24.54 & 107 & 24.54 & & \\
\hline $45-54$ & 171 & 39.22 & 171 & 39.22 & & \\
\hline $55-64$ & 95 & 21.79 & 95 & 21.79 & & \\
\hline $65+$ & 9 & 2.06 & 9 & 2.06 & & \\
\hline Sex & & & & & N/A & N/A \\
\hline Male & 55 & 12.61 & 55 & 12.61 & & \\
\hline Female & 381 & 87.39 & 381 & 87.39 & & \\
\hline Region & & & & & N/A & N/A \\
\hline Northeast & 53 & 12.16 & 53 & 12.16 & & \\
\hline North Central & 124 & 28.44 & 124 & 28.44 & & \\
\hline South & 166 & 38.07 & 166 & 38.07 & & \\
\hline West & 93 & 21.10 & 93 & 21.10 & & \\
\hline Unknown & 1 & 0.23 & 1 & 0.23 & & \\
\hline Year of index date & & & & & N/A & N/A \\
\hline 2004 & 66 & 15.14 & 66 & 15.14 & & \\
\hline 2005 & 109 & 25.00 & 109 & 25.00 & & \\
\hline 2006 & 109 & 25.00 & 109 & 25.00 & & \\
\hline 2007 & 129 & 29.59 & 129 & 29.59 & & \\
\hline 2008 & 23 & 5.28 & 23 & 5.28 & & \\
\hline ITT DMT & & & & & N/A & N/A \\
\hline Avonex & 97 & 22.25 & 97 & 22.25 & & \\
\hline Betaseron & 51 & 11.70 & 51 & 11.70 & & \\
\hline Copaxone & 198 & 45.41 & 198 & 45.41 & & \\
\hline Rebif & 78 & 17.89 & 78 & 17.89 & & \\
\hline Tysabri & 12 & 2.75 & 12 & 2.75 & & \\
\hline \multicolumn{7}{|l|}{ Other characteristics } \\
\hline \multicolumn{7}{|l|}{ Insurance status } \\
\hline Comprehensive & 58 & 13.30 & 43 & 9.86 & 2.52 & 0.112 \\
\hline Health maintenance org & 80 & 18.35 & 90 & 20.64 & 0.73 & 0.393 \\
\hline Point-of-service & 99 & 22.71 & 58 & 13.30 & 13.06 & $<0.001$ \\
\hline Preferred provider org & 191 & 43.81 & 226 & 51.83 & 5.63 & 0.018 \\
\hline Other & 8 & 1.83 & 19 & 4.36 & 4.63 & 0.032 \\
\hline Copayment with ITT drug & 370 & 84.86 & 340 & 77.98 & 6.82 & 0.009 \\
\hline Mail order for ITT drug & 145 & 33.26 & 119 & 27.29 & 3.67 & 0.055 \\
\hline \multicolumn{7}{|l|}{ Preperiod Charlson score } \\
\hline 0 & 331 & 75.92 & 271 & 61.16 & 19.31 & $<0.001$ \\
\hline $1-2$ & 93 & 21.33 & 132 & 30.28 & 9.11 & 0.003 \\
\hline $3+$ & 12 & 2.75 & 33 & 7.57 & 10.33 & $<0.001$ \\
\hline \multicolumn{7}{|l|}{ Preperiod disability proxies } \\
\hline Muscoloskeletal & 181 & 41.51 & 262 & 60.09 & 30.10 & $<0.001$ \\
\hline Medical & 137 & 31.42 & 210 & 48.17 & 25.51 & $<0.001$ \\
\hline
\end{tabular}




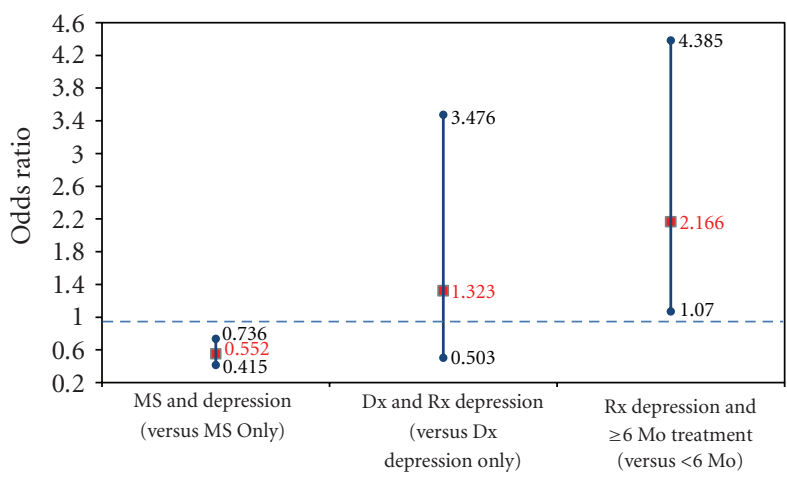

Odds ratio odds ratio (in red) with 95\% confidence intervals Patients with MS and depression, compared to those with MS only, were $44.8 \%$ (100-0.552) less likely to achieve a MPR threshold of at least $80 \%$ with their MS medication. Patients with comorbid depression who were treated with an antidepressant for at least 6 months, compared to those treated with an antidepressant for $<6$ months, were $116.6 \%$ (2.16-1) more likely to achieve an MPR threshold of at Least $80 \%$.

FIgURE 2: Impact of depression on probability of achieving MPR threshold of 0.80 .

22.71\%; $P<0.01$ ), but significantly more likely to be insured via a preferred provider organization (51.83\% versus $43.81 \% ; P=0.02)$ or to have "other" insurance $(4.36 \%$ versus $1.83 \% ; P=0.03)$. In addition, patients with comorbid depression were less likely to have a copayment associated with their index ITT medication prescription $(77.98 \%$ versus $84.86 \% ; P=0.01)$. Comparing patients with MS only to those with MS and depression revealed significant differences in both general health and functional limitations between the two groups, with patients with comorbid depression generally in poorer health. For example, patients diagnosed with comorbid depression were less likely to have a Charlson score of 0 (62.18\% versus $75.92 \% ; P<0.01)$ and more likely to have a Charlson score of $1-2$ (30.28\% versus $21.33 \%$; $P<$ $0.01)$ or 3 or more $(7.57 \%$ versus $2.75 \% ; P<0.01)$. Similarly, patients with comorbid depression were significantly more likely to be diagnosed with a musculoskeletal limitation (60.09\% versus $41.51 \% ; P<0.01)$ or medical limitation (48.17\% versus $31.42 \% ; P<0.01$ ).

In contrast to the comparison of patients with MS Only to those with MS and depression, comparisons between patients with MS and comorbid depression treated and untreated with antidepressants revealed few differences. For example, Table 2 compared patients diagnosed with depression who did not receive an antidepressant to those patients who were both diagnosed with depression and received at least one prescription for an antidepressant. There were no statistically significant differences found among these two groups when comparing insurance status, copayment or mail order status, CCI, or disability proxies (Table 2). Similarly, comparing patients who received at least 6 months of an antidepressant to individuals who received less than 6 months of an antidepressant (Table 3 ) revealed few differences between the two groups.
3.2. Results: Ordinary Least Squares Regressions. Table 4 shows the results of the ordinary least squares regression models that examine the association between comorbid depression and patient adherence to ITT DMT for MS. Comparing patients with Ms only to those with MS and depression, results indicate that, after controlling for patient insurance type, characteristics of initial DMT dispensed, patient general health, and functional limitations, patients with comorbid depression had a 10\% lower MPR score than those without comorbid depression (coefficient $=-0.10$; $P<0.01)$. Results also reveal that patients with healthmaintenance insurance, compared to those with a preferred provider organization had significantly lower medication adherence (coefficient $=-0.10 ; P=0.02$ ) while patients who had a copayment associated with their ITT DMT had significantly higher medication adherence $($ coefficient $=0.06$; $P=0.05)$.

Comparing patients diagnosed with depression but untreated to those both diagnosed and treated with an antidepressant revealed no significant association between treatment for depression with antidepressants and patient adherence to their MS therapy (coefficient $=0.07 ; P=0.52$ ). However, patients treated with an antidepressant for at least 6 months compared to those treated for less than 6 months have, on average, a 12-point higher MPR (coefficient $=0.12$; $P=0.05$ ) after controlling for insurance type, characteristics of initial DMT dispensed, and patient general health and functional limitation.

3.3. Results: Logistic Models. In addition to examining the impact of depression on MPR levels, Figure 2 examines how comorbid depression affects the probability of achieving a threshold of at least $80 \%$. These results are generally consistent with the results from the ordinary least squares regression which examine MPR as a continuous variable. Specifically, results from these multivariate logistic models indicate that patients who were depressed (based upon either diagnosis or diagnosis and receipt of antidepressant) were $45 \%$ less likely to achieve an MPR threshold of $80 \%$ (odds ratio (OR) 0.55; 95\% confidence interval (CI) $0.42-$ 0.74). An examination of the subset of patients with both MS and depression revealed that those treated with an antidepressant, irrespective of length of treatment, had no significant difference in the likelihood of achieving an MPR threshold of $80 \%(\mathrm{OR}=1.32 ; 95 \%$ CI $0.50-3.48)$. However, those treated with an antidepressant for at least 6 months were twice as likely to reach an MPR threshold of $80 \%$ with their DMT, compared to those treated with an antidepressant for less than 6 months $(\mathrm{OR}=2.17$; 95\% CI 1.07-4.39).

3.4. Robustness of Results. As a test of the robustness of the results, alternative specifications were also examined. First, we constructed an alternative model that allowed for patient age, sex, region of residence, and ITT medication to be explicitly included in the multivariate analyses. Second, as discussed above, adherence was also proxied by the proportion of days covered. Third, to examine the impact of the relatively small sample when comparing those patients 
TABLE 2: Patient characteristics: MS patients diagnosed with depression but untreated compared to MS patients diagnosed with depression who received an antidepressant.

\begin{tabular}{|c|c|c|c|c|c|c|}
\hline \multirow{2}{*}{ Characteristic } & \multicolumn{2}{|c|}{ Dx Depression $(N=38)$} & \multicolumn{2}{|c|}{ Dx \& Rx Depression $(N=38)$} & \multirow{2}{*}{$\chi^{2}$ statistic } & \multirow{2}{*}{$P$ value } \\
\hline & $N$ & $\%$ & $N$ & $\%$ & & \\
\hline \multicolumn{7}{|l|}{ Characteristics matched on } \\
\hline Age & & & & & N/A & N/A \\
\hline$<35$ & 1 & 2.63 & 1 & 2.63 & & \\
\hline $35-44$ & 8 & 21.04 & 8 & 21.04 & & \\
\hline $45-54$ & 19 & 50.00 & 19 & 50.00 & & \\
\hline $55-64$ & 10 & 26.32 & 10 & 26.32 & & \\
\hline $65+$ & 0 & 0 & 0 & 0 & & \\
\hline Sex & & & & & N/A & N/A \\
\hline Male & 2 & 5.26 & 2 & 5.26 & & \\
\hline Female & 36 & 94.74 & 36 & 94.74 & & \\
\hline Region & & & & & N/A & N/A \\
\hline Northeast & 3 & 7.89 & 3 & 7.89 & & \\
\hline North Central & 13 & 34.21 & 13 & 34.21 & & \\
\hline South & 12 & 31.58 & 12 & 31.58 & & \\
\hline West & 10 & 26.32 & 10 & 26.32 & & \\
\hline Unknown & 0 & 0 & 0 & 0 & & \\
\hline Year of index date & & & & & N/A & N/A \\
\hline 2004 & 6 & 15.79 & 6 & 15.79 & & \\
\hline 2005 & 11 & 28.95 & 11 & 28.95 & & \\
\hline 2006 & 10 & 26.32 & 10 & 26.32 & & \\
\hline 2007 & 10 & 26.32 & 10 & 26.32 & & \\
\hline 2008 & 1 & 2.63 & 1 & 2.63 & & \\
\hline ITT DMT & & & & & N/A & N/A \\
\hline Avonex & 12 & 31.58 & 12 & 31.58 & & \\
\hline Betaseron & 2 & 5.26 & 2 & 5.26 & & \\
\hline Copaxone & 19 & 50.00 & 19 & 50.00 & & \\
\hline Rebif & 4 & 10.53 & 4 & 10.53 & & \\
\hline Tysabri & 1 & 2.63 & 1 & 2.63 & & \\
\hline \multicolumn{7}{|l|}{ Other characteristics } \\
\hline \multicolumn{7}{|l|}{ Insurance status } \\
\hline Comprehensive & 6 & 15.79 & 4 & 13.16 & 0.11 & 0.744 \\
\hline Health maintenance org & 5 & 13.16 & 9 & 23.68 & 1.40 & 0.236 \\
\hline Point-of-service & 6 & 15.79 & 7 & 18.42 & 0.93 & 0.761 \\
\hline Preferred provider org & 20 & 52.63 & 16 & 42.11 & 0.84 & 0.358 \\
\hline Other & 1 & 2.63 & 1 & 2.63 & N/A & N/A \\
\hline Copayment with ITT drug & 29 & 76.32 & 29 & 76.32 & N/A & N/A \\
\hline Mail order for ITT drug & 13 & 34.21 & 10 & 26.32 & 0.56 & 0.454 \\
\hline \multicolumn{7}{|l|}{ Preperiod Charlson score } \\
\hline 0 & 23 & 60.53 & 21 & 55.26 & 0.22 & 0.642 \\
\hline $1-2$ & 11 & 28.95 & 15 & 39.47 & 0.93 & 0.334 \\
\hline $3+$ & 4 & 10.53 & 2 & 5.26 & 0.72 & 0.395 \\
\hline \multicolumn{7}{|l|}{ Preperiod disability proxies } \\
\hline Muscoloskeletal & 25 & 65.79 & 24 & 63.16 & 0.06 & 0.811 \\
\hline Medical & 16 & 42.11 & 18 & 47.37 & 0.22 & 0.645 \\
\hline
\end{tabular}


TABle 3: Patient characteristics: MS patients treated with antidepressants for at least 6 months compared to MS patients treated with antidepressants for less than 6 months.

\begin{tabular}{|c|c|c|c|c|c|c|}
\hline \multirow{2}{*}{ Characteristic } & \multicolumn{2}{|c|}{ Treated less than guidelines $(N=71)$} & \multicolumn{2}{|c|}{ Treated according to guidelines $(N=71)$} & \multirow{2}{*}{$\chi^{2}$ statistic } & \multirow{2}{*}{$P$ value } \\
\hline & $N$ & $\%$ & $N$ & $\%$ & & \\
\hline \multicolumn{7}{|l|}{ Characteristics matched on } \\
\hline Age & & & & & N/A & N/A \\
\hline$<35$ & 5 & 7.04 & 5 & 7.04 & & \\
\hline $35-44$ & 17 & 23.94 & 17 & 23.94 & & \\
\hline $45-54$ & 32 & 45.07 & 32 & 45.07 & & \\
\hline $55-64$ & 17 & 23.94 & 17 & 23.94 & & \\
\hline $65+$ & 0 & 0 & 0 & 0 & & \\
\hline Sex & & & & & N/A & N/A \\
\hline Male & 1 & 1.41 & 1 & 1.41 & & \\
\hline Female & 70 & 98.59 & 70 & 98.59 & & \\
\hline Region & & & & & N/A & N/A \\
\hline Northeast & 5 & 7.04 & 5 & 7.04 & & \\
\hline North Central & 21 & 29.58 & 21 & 29.58 & & \\
\hline South & 30 & 42.25 & 30 & 42.25 & & \\
\hline West & 15 & 21.13 & 15 & 21.13 & & \\
\hline Unknown & 0 & 0 & 0 & 0 & & \\
\hline Year of index date & & & & & N/A & N/A \\
\hline 2004 & 6 & 8.45 & 6 & 8.45 & & \\
\hline 2005 & 17 & 23.94 & 17 & 23.94 & & \\
\hline 2006 & 15 & 21.13 & 15 & 21.13 & & \\
\hline 2007 & 28 & 39.44 & 28 & 39.44 & & \\
\hline 2008 & 5 & 7.04 & 5 & 7.04 & & \\
\hline ITT DMT & & & & & $\mathrm{N} / \mathrm{A}$ & N/A \\
\hline Avonex & 11 & 15.49 & 11 & 15.49 & & \\
\hline Betaseron & 3 & 4.23 & 3 & 4.23 & & \\
\hline Copaxone & 44 & 61.97 & 44 & 61.97 & & \\
\hline Rebif & 13 & 18.31 & 13 & 18.31 & & \\
\hline Tysabri & 0 & 9 & 0 & 9 & & \\
\hline \multicolumn{7}{|l|}{ Other characteristics } \\
\hline \multicolumn{7}{|l|}{ Insurance status } \\
\hline Comprehensive & 6 & 8.45 & 6 & 8.45 & N/A & $\mathrm{N} / \mathrm{A}$ \\
\hline Health maintenance org & 16 & 22.54 & 10 & 14.08 & 1.70 & 0.193 \\
\hline Point-of-service & 10 & 14.08 & 6 & 8.45 & 1.13 & 0.288 \\
\hline Preferred provider org & 39 & 54.93 & 45 & 63.38 & 1.05 & 0.306 \\
\hline Other & 0 & 0 & 4 & 5.63 & 4.12 & 0.043 \\
\hline Copayment with ITT drug & 61 & 85.92 & 54 & 76.06 & 2.24 & 0.134 \\
\hline Mail order for ITT drug & 23 & 32.39 & 22 & 30.99 & 0.03 & 0.857 \\
\hline \multicolumn{7}{|l|}{ Preperiod Charlson score } \\
\hline 0 & 45 & 63.38 & 40 & 56.34 & 0.73 & 0.392 \\
\hline $1-2$ & 20 & 28.17 & 26 & 36.62 & 1.16 & 0.282 \\
\hline $3+$ & 6 & 8.45 & 5 & 7.04 & 0.09 & 0.754 \\
\hline \multicolumn{7}{|l|}{ Preperiod disability proxies } \\
\hline Muscoloskeletal & 46 & 64.79 & 48 & 67.61 & 0.13 & 0.723 \\
\hline Medical & 35 & 49.30 & 42 & 59.15 & 1.39 & 0.238 \\
\hline
\end{tabular}


TABLE 4: Ordinary least squares regression results dependent variable: MPR.

\begin{tabular}{|c|c|c|c|c|c|c|}
\hline \multirow{2}{*}{ Variable } & \multicolumn{2}{|c|}{ MS Only versus MS \& Depression } & \multicolumn{2}{|c|}{$\begin{array}{l}\text { Dx Depression versus Dx \& Rx } \\
\text { Depression }\end{array}$} & \multicolumn{2}{|c|}{$\begin{array}{c}\mathrm{Rx} \text { Depression- } \geq 6 \mathrm{Mo} \\
\text { Antidepressants versus }<6 \mathrm{Mo}\end{array}$} \\
\hline & Coeff & $P$ value & Coeff & $P$ value & Coeff & $P$ value \\
\hline Intercept & 0.71 & $<0.001$ & 0.64 & 0.047 & 0.47 & $<0.001$ \\
\hline \multicolumn{7}{|l|}{ Insurance type $\mathrm{a}^{\mathrm{a}}$} \\
\hline HMO & -0.10 & 0.022 & -0.14 & 0.509 & -0.16 & 0.200 \\
\hline POS & -0.02 & 0.665 & -0.07 & 0.731 & 0.02 & 0.901 \\
\hline $\mathrm{PPO}$ & 0.01 & 0.890 & 0.12 & 0.491 & 0.02 & 0.860 \\
\hline Other & -0.002 & 0.974 & -0.27 & 0.523 & 0.08 & 0.693 \\
\hline \multicolumn{7}{|l|}{ Medication dispensing } \\
\hline Copayment & 0.06 & 0.047 & -0.06 & 0.680 & 0.10 & 0.213 \\
\hline Mail order & 0.05 & 0.051 & 0.02 & 0.909 & 0.08 & 0.216 \\
\hline \multicolumn{7}{|l|}{ Preperiod Charlson score ${ }^{\mathrm{b}}$} \\
\hline $1-2$ & 0.01 & 0.738 & 0.09 & 0.521 & 0.06 & 0.379 \\
\hline $3+$ & -0.04 & 0.467 & 0.04 & 0.853 & 0.09 & 0.422 \\
\hline \multicolumn{7}{|l|}{ Preperiod ADL proxies } \\
\hline Musculoskeletal & 0.02 & 0.440 & -0.03 & 0.828 & 0.06 & 0.294 \\
\hline Medical & 0.02 & 0.448 & 0.10 & 0.419 & -0.01 & 0.889 \\
\hline \multicolumn{7}{|l|}{ Preperiod depression } \\
\hline Any & $-0.10^{c}$ & $<0.001$ & - & - & - & - \\
\hline$D x \& R x$ & - & - & $0.07^{\mathrm{d}}$ & 0.518 & - & - \\
\hline $\mathrm{Rx}$ for at least 6 months & - & - & - & - & $0.12^{\mathrm{e}}$ & 0.049 \\
\hline Adjusted $R^{2}$ & \multicolumn{2}{|c|}{0.05} & \multicolumn{2}{|c|}{0.08} & \multicolumn{2}{|c|}{0.06} \\
\hline
\end{tabular}

${ }^{a}$ Reference group: Comprehensive insurance.

${ }^{b}$ Reference group: Charlson score of 0 .

${ }^{\mathrm{c}}$ Reference group: patients with MS and no diagnosis of depression or receipt of an antidepressant.

${ }^{\mathrm{d}}$ Reference group: patients diagnosed with depression who did not receive an antidepressant.

${ }^{\text {e}}$ Reference group: patients diagnosed with depression who were treated with an antidepressant for less than 6 months.

diagnosed with depression but untreated to those diagnosed with depression who received an antidepressant, the analysis also examined this subgroup without the reduction of sample size that is the result of matching the two cohorts; none of these modifications resulted in any significant changes in the results reported.

3.5. Discussion. Focused on a population consisting entirely of patients with MS, this analysis was designed to determine the impact of comorbid depression on adherence to DMT. This study also sought to determine the impact of antidepressant therapy on adherence to DMT among patients with MS and comorbid depression. This analysis produced several noteworthy results.

First, patients diagnosed with comorbid depression were sicker than those without comorbid depression, as indicated by their likelihood of a higher score on the Charlson Comorbidity Index (see Table 1), as well as their greater likelihood of having a musculoskeletal limitation $(60.09 \%$ versus $41.51 \%$; $P<0.01)$ or any medical limitation $(48.17 \%$ versus $31.42 \%$; $P<0.01)$. This association between comorbid depression and a larger burden of illness is consistent with research on other disease states, research which has shown that patients with diabetes and comorbid depression have more complications relative to diabetic patients without depression [30] and that heart disease patients with comorbid depression have greater morbidity and mortality relative to their counterparts without depression [31-37].

Second, patients with MS and comorbid depression had, on average, a 10-point lower MPR and were approximately half $(45 \%)$ as likely to reach a threshold MPR level of $80 \%$ relative to the matched cohort with MS without depression. The strong association between depression and difficulty with adherence was expected and was consistent with the findings of earlier research conducted among patients with MS [10, 13]. It also was consistent with research showing the negative effect of comorbid depression on adherence to therapy for other diseases, including hypertension [38], diabetes $[10,11]$, and heart disease $[39,40]$. While previous research among patients with MS has shown comorbid depression to be associated with adherence difficulties [10, 13], our research expands the literature by quantifying the adherence gap between those MS patients with comorbid depression compared to those without depression.

Third, results from this analysis revealed no significant difference in adherence to DMT therapy when comparing 
patients diagnosed with depression that were not treated with an antidepressant to those both diagnosed and treated for depression. However, among patients with comorbid depression, those who received at least a 6-month supply of antidepressants had, on average, a 10-point higher MPR and were more than twice as likely to reach an MPR threshold of $80 \%$ relative to those who filled their antidepressant medication prescription for less than a 6-month period. Significantly, in our study, merely having a prescription for an antidepressant had no significant impact on DMT adherence. Instead, the positive impact of antidepressant therapy on DMT adherence was observed only among patients whose antidepressant therapy extended for at least 6 months. This finding supports previous research conducted among patients diagnosed with diabetes, which showed that antidepressant therapy improved adherence to diabetes treatment and medical outcomes [41]. However, caution must be taken when interpreting this finding since results are also consistent with the hypothesis that patients who are adherent to their antidepressant therapy are generally more compliant at taking their medications and hence, more likely to be adherent to their DMT as well.

3.6. Limitations. As with any research, the findings presented here should be interpreted within the context of the limitations of the study design. First, this analysis was conducted using an administrative claims database and included only patients with medical and outpatient prescription drug benefit coverage. The results, therefore, may not generalize well to other populations. Second, it is less rigorous to rely upon diagnostic codes rather than formal diagnostic assessment for identifying patients or for measuring patient general health and functional limitations. For example, our reliance on diagnostic claims and the criteria utilized for identifying patients with depression resulted in approximately $6 \%$ of our MS cohort with comorbid depression while previous research has shown that up to $50 \%$ of MS patients experience comorbid depression [14, 15]. Third, this analysis used MPRs and PDCs to measure medication adherence. Such measures are unable to identify actual drug-taking behavior or patient adherence to filling written prescriptions. Fourth, the database was unable to capture other information, such as patient personality type, caregiver support, and type of MS, that may also impact outcomes. Finally, this study focuses on a subset of individuals who were diagnosed with depression prior to receipt of a DMT for MS. The results, therefore, may not apply to individuals who are diagnosed with depression subsequent to initiation on treatment for MS.

\section{Conclusions}

In conclusion, this study indicates that those with MS and comorbid depression are sicker and approximately only half as likely to be adherent to their disease-modifying therapy relative to patients with MS without comorbid depression. This analysis also shows were no significant difference in DMT adherence when comparing patients diagnosed with depression but not treated with an antidepressant to those both diagnosed and treated for depression. However, those with MS and comorbid depression who take antidepressants for at least 6 months are more than twice as likely to be adherent to their DMT relative to those with MS and comorbid depression who do not remain on antidepressant therapy for at least 6 months. Future work should focus on examining these results with alternative data sources and improving our understanding of the relationship between adherence to antidepressant therapy and adherence to DMT.

\section{Acknowledgment}

The authors would like to thank Patricia Platt for help in the writing of the paper.

\section{References}

[1] A. M. Courtney, K. Treadaway, G. Remington, and E. Frohman, "Multiple sclerosis," Medical Clinics of North America, vol. 93, no. 2, pp. 451-476, ix-x, 2009.

[2] National Multiple Sclerosis Society, "FAQs about MS: National MS Society," 2009, http://www.nationalmssociety.org/aboutmultiple-sclerosis/what-we-know-about-ms/FAQs-about-MS/ index.aspx\#howmany.

[3] H. Naci, R. Fleurence, J. Birt, and A. Duhig, "Economic burden of multiple sclerosis: a systematic review of the literature," PharmacoEconomics, vol. 28, no. 5, pp. 363-379, 2010.

[4] J. A. O'Brien, A. J. Ward, A. R. Patrick, and J. Caro, "Cost of managing an episode of relapse in multiple sclerosis in the United States," BMC Health Services Research, vol. 3, no. 1, p. 17, 2003.

[5] J. D. Prescott, S. Factor, M. Pill, and G. W. Levi, "Descriptive analysis of the direct medical costs of multiple sclerosis in 2004 using administrative claims in a large nationwide database," Journal of Managed Care Pharmacy, vol. 13, no. 1, pp. 44-52, 2007.

[6] K. Whetten-Goldstein, F. A. Sloan, L. B. Goldstein, and E. D. Kulas, "A comprehensive assessment of the cost of multiple sclerosis in the United States," Multiple Sclerosis, vol. 4, no. 5, pp. 419-425, 1998.

[7] National Multiple Sclerosis Society, "Multiple Sclerosis: Just the Facts," 2009, http://www.nationalmssociety.org/aboutmultiple-sclerosis/ what-we-know-about-ms/ what-is-ms/ index.aspx.

[8] M. J. Tullman, F. D. Lublin, and A. E. Miller, "Immunotherapy of multiple sclerosis-current practice and future directions," Journal of Rehabilitation Research and Development, vol. 39, no. 2, pp. 273-285, 2002.

[9] C. Van Houtven, M. Weinberger, and T. Carey, "Implications of nonadherence for economic evaluation and health policy," in Patient Treatment Adherence: Concepts, Interventions, and Measurement, pp. 421-451, Routledge, London, UK, 2005.

[10] J. M. Bruce, L. M. Hancock, P. Arnett, and S. Lynch, "Treatment adherence in multiple sclerosis: association with emotional status, personality, and cognition," Journal of Behavioral Medicine, vol. 33, no. 3, pp. 219-227, 2010.

[11] J. M. Bruce, L. M. Hancock, and S. G. Lynch, "Objective adherence monitoring in multiple sclerosis: initial validation and association with self-report," Multiple Sclerosis, vol. 16, no. 1, pp. 112-120, 2010. 
[12] A. Lugaresi, "Addressing the need for increased adherence to multiple sclerosis therapy: can delivery technology enhance patient motivation?" Expert Opinion on Drug Delivery, vol. 6, no. 9, pp. 995-1002, 2009.

[13] K. Treadaway, G. Cutter, A. Salter et al., "Factors that influence adherence with disease-modifying therapy in MS," Journal of Neurology, vol. 256, no. 4, pp. 568-576, 2009.

[14] R. A. Marrie, R. Horwitz, G. Cutter, T. Tyry, D. Campagnolo, and T. Vollmer, "The burden of mental comorbidity in multiple sclerosis: frequent, underdiagnosed, and undertreated," Multiple Sclerosis, vol. 15, no. 3, pp. 385-392, 2009.

[15] A. D. Sadovnick, R. A. Remick, J. Allen et al., "Depression and multiple sclerosis," Neurology, vol. 46, no. 3, pp. 628-632, 1996.

[16] R. B. Schiffer and H. B. Babigian, "Behavioral disorders in multiple sclerosis, temporal lobe epilepsy, and amyotrophic lateral sclerosis: an epidemiologic study," Archives of Neurology, vol. 41, no. 10, pp. 1067-1069, 1984.

[17] J. I. Ivanova, H. G. Birnbaum, Y. Kidolezi, G. Subramanian, S. A. Khan, and M. D. Stensland, "Direct and indirect costs of employees with treatment-resistant and non-treatmentresistant major depressive disorder," Current Medical Research and Opinion, vol. 26, no. 10, pp. 2475-2484, 2010.

[18] K. Lang, J. L. Meyers, J. R. Korn et al., "Medication adherence and hospitalization among patients with schizophrenia treated with antipsychotics," Psychiatric Services, vol. 61, no. 12, pp. 1239-1247, 2010.

[19] A. Qaseem, V. Snow, T. D. Denberg, M. A. Forciea, and D. K. Owens, "Using second-generation antidepressants to treat depressive disorders: a clinical practice guideline from the American College of Physicians," Annals of Internal Medicine, vol. 149, no. 10, pp. 725-733, 2008.

[20] J. A. Cramer, A. Roy, A. Burrell et al., "Medication compliance and persistence: terminology and definitions," Value in Health, vol. 11, no. 1, pp. 44-47, 2008.

[21] J. Cooper, L. Hall, A. Penland, A. Krueger, and J. May, "Measuring medication adherence," Population Health Management, vol. 12, no. 1, pp. 25-30, 2009.

[22] J. I. Ivanova, H. G. Birnbaum, M. Hsieh et al., "Adherence to inhaled corticosteroid use and local adverse events in persistent asthma," The American Journal of Managed Care, vol. 14, no. 12, pp. 801-809, 2008.

[23] B. C. Martin, E. K. Wiley-Exley, S. Richards, M. E. Domino, T. S. Carey, and B. L. Sleath, "Contrasting measures of adherence with simple drug use, medication switching, and therapeutic duplication," Annals of Pharmacotherapy, vol. 43, no. 1, pp. 3644, 2009.

[24] H. Florez, J. Luo, S. Castillo-Florez et al., "Impact of metformin-induced gastrointestinal symptoms on quality of life and adherence in patients with type 2 diabetes," Postgraduate Medicine, vol. 122, no. 2, pp. 112-120, 2010.

[25] R. L. Sedjo and S. Devine, "Predictors of non-adherence to aromatase inhibitors among commercially insured women with breast cancer," Breast Cancer Research and Treatment, vol. 125, no. 1, pp. 191-200, 2011.

[26] W. D'Hoore, C. Sicotte, and C. Tilquin, "Risk adjustment in outcome assessment: the Charlson comorbidity index," Methods of Information in Medicine, vol. 32, no. 5, pp. 382387, 1993.

[27] R. A. Deyo, D. C. Cherkin, and M. A. Ciol, "Adapting a clinical comorbidity index for use with ICD-9-CM administrative databases," Journal of Clinical Epidemiology, vol. 45, no. 6, pp. 613-619, 1992.
[28] V. Sundararajan, H. Quan, P. Halfan et al., "Cross-national comparative performance of three versions of the ICD-10 Charlson index," Medical Care, vol. 45, no. 12, pp. 1210-1215, 2007.

[29] L. Chan, A. Shumway-Cook, K. M. Yorkston, M. A. Ciol, B. J. Dudgeon, and J. M. Hoffman, "Design and validation of a methodology using the International Classification of Diseases, 9th Revision, to identify secondary conditions in people with disabilities," Archives of Physical Medicine and Rehabilitation, vol. 86, no. 5, pp. 1065-1069, 2005.

[30] M. de Groot, R. Anderson, K. E. Freedland, R. E. Clouse, and P. J. Lustman, "Association of depression and diabetes complications: a meta-analysis," Psychosomatic Medicine, vol. 63, no. 4, pp. 619-630, 2001.

[31] J. C. Barefoot, B. H. Brummett, M. J. Helms, D. B. Mark, I. C. Siegler, and R. B. Williams, "Depressive symptoms and survival of patients with coronary artery disease," Psychosomatic Medicine, vol. 62, no. 6, pp. 790-795, 2000.

[32] M. M. Burg, M. C. Benedetto, and R. Soufer, "Depressive symptoms and mortality two years after coronary artery bypass graft surgery (CABG) in men," Psychosomatic Medicine, vol. 65, no. 4, pp. 508-510, 2003.

[33] N. Frasure-Smith and F. Lespérance, "Depression and other psychological risks following myocardial infarction," Archives of General Psychiatry, vol. 60, no. 6, pp. 627-636, 2003.

[34] M. W. Kaufmann, J. P. Fitzgibbons, E. J. Sussman et al., "Relation between myocardial infarction, depression, hostility, and death," The American Heart Journal, vol. 138, no. 3, part 1, pp. 549-554, 1999.

[35] K. H. Ladwig, M. Kieser, J. König, G. Breithardt, and M. Borggrefe, "Affective disorders and survival after acute myocardial infarction. Results from the post-infarction late potential study," The European Heart Journal, vol. 12, no. 9, pp. 959964, 1991.

[36] F. Lespérance, N. Frasure-Smith, M. Juneau, and P. Théroux, "Depression and 1-year prognosis in unstable angina," Archives of Internal Medicine, vol. 160, no. 9, pp. 1354-1360, 2000.

[37] F. Lespérance, N. Frasure-Smith, M. Talajic, and M. G. Bourassa, "Five-year risk of cardiac mortality in relation to initial severity and one-year changes in depression symptoms after myocardial infarction," Circulation, vol. 105, no. 9, pp. 10491053, 2002.

[38] C. M. Eze-Nliam, B. D. Thombs, B. B. Lima, C. G. Smith, and R. C. Ziegelstein, "The association of depression with adherence to antihypertensive medications: a systematic review," Journal of Hypertension, vol. 28, no. 9, pp. 1785-1795, 2010.

[39] J. B. Garner, "Problems of nonadherence in cardiology and proposals to improve outcomes," The American Journal of Cardiology, vol. 105, no. 10, pp. 1495-1501, 2010.

[40] A. Gehi, D. Haas, S. Pipkin, and M. A. Whooley, "Depression and medication adherence in outpatients with coronary heart disease," Archives of Internal Medicine, vol. 165, no. 21, pp. 2508-2513, 2005.

[41] H. Abrahamian, P. Hofmann, R. Prager, and H. Toplak, "Diabetes mellitus and co-morbid depression: treatment with milnacipran results in significant improvement of both diseases (results from the Austrian MDDM study group)," Neuropsychiatric Disease and Treatment, vol. 5, no. 1, pp. 261266, 2009. 


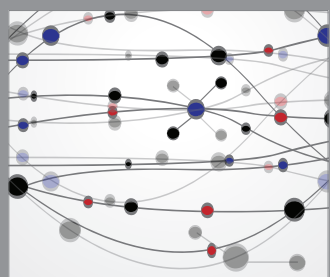

The Scientific World Journal
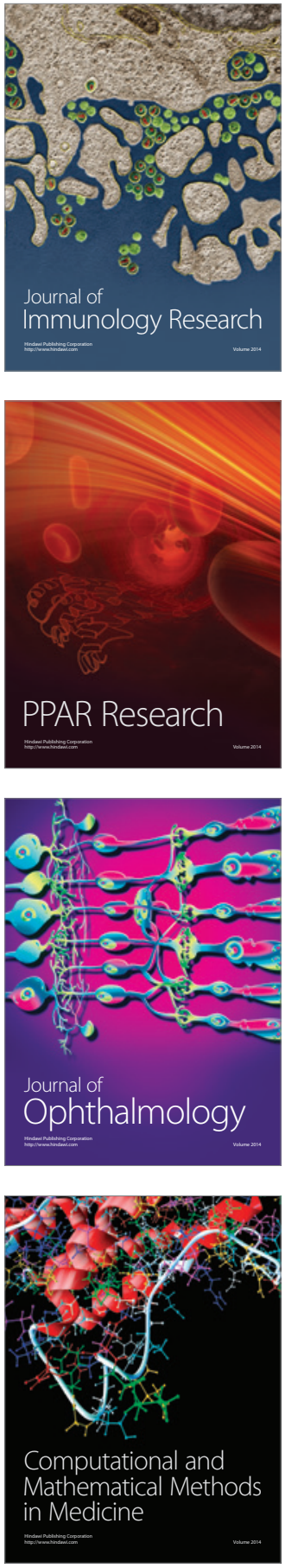

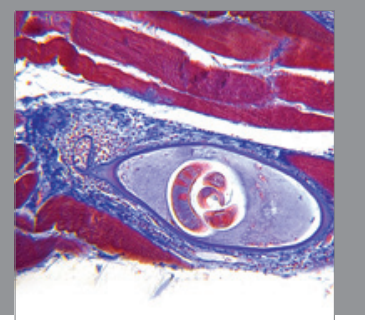

Gastroenterology

Research and Practice
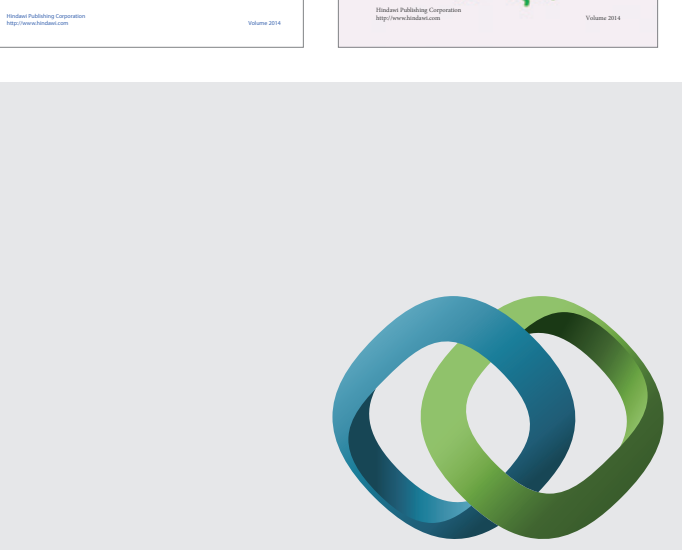

\section{Hindawi}

Submit your manuscripts at

http://www.hindawi.com
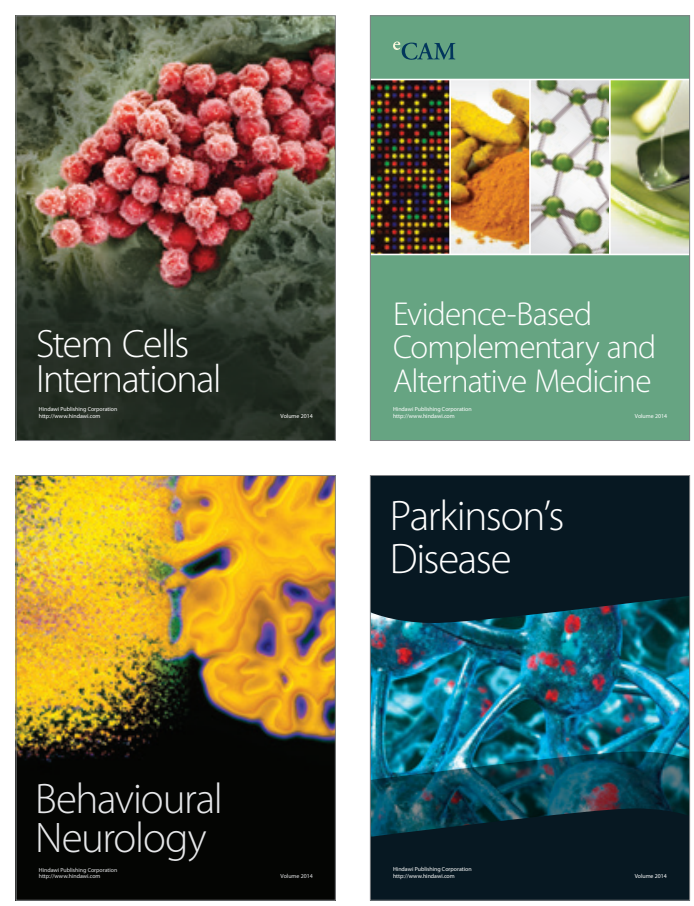

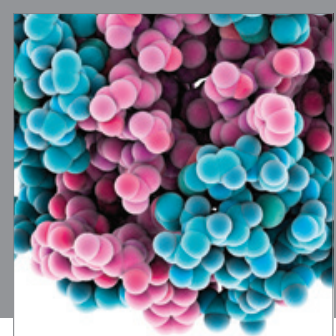

Journal of
Diabetes Research

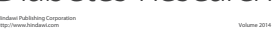

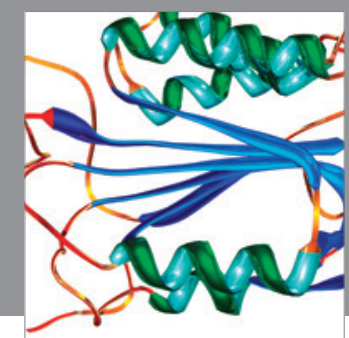

Disease Markers
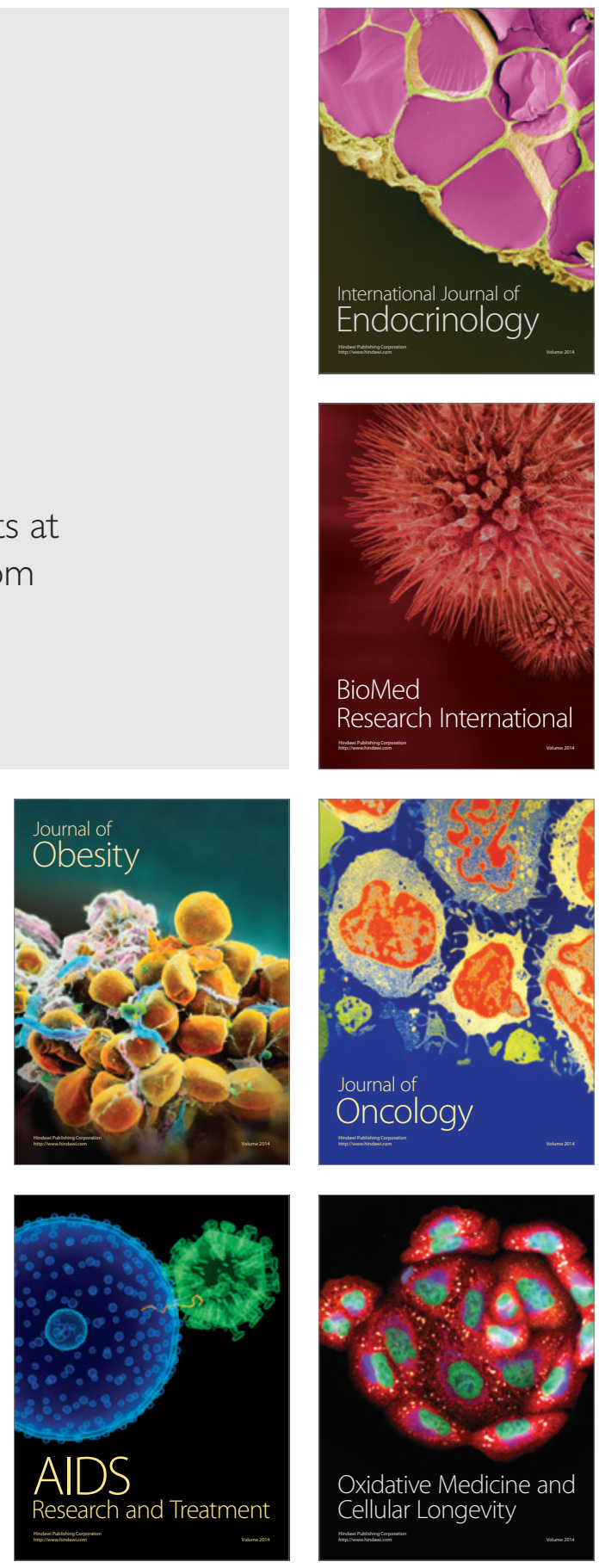